\begin{tabular}{|c|l|}
\hline Title & $\begin{array}{l}\text { Fabrication and characterization of freestanding GaA s/A IGaA s core shell nanowires and A IGaA s nanotubes by using } \\
\text { selective area metal organic vapor phase epitaxy }\end{array}$ \\
\hline Author(s) & Noborisaka, J.; Motohisa, J.; Hara, S.; Fukui, T. \\
\hline Citation & $\begin{array}{l}\text { A pplied Physics Letters, 87(9) } \\
\text { https://doi.org/10.1063/2035332 }\end{array}$ \\
\hline Issue Date & 2005-08-29 \\
\hline Doc URL & http://hdl.handle.net/2115/5503 \\
\hline Rights & Copyright $\odot 2005$ A merican Institute of Physics \\
\hline Type & article \\
\hline File Information & APL 87(9).pdf \\
\hline
\end{tabular}

Instructions for use 


\title{
Fabrication and characterization of freestanding GaAs/AIGaAs core-shell nanowires and AIGaAs nanotubes by using selective-area metalorganic vapor phase epitaxy
}

\author{
J. Noborisaka, ${ }^{\text {a) }}$ J. Motohisa, S. Hara, and T. Fukui \\ Graduate School of Information Science and Technology and Research Center for Integrated Quantum \\ Electronics, Hokkaido University, North 14 West 9, Sapporo 060-0814, Japan
}

(Received 14 March 2005; accepted 7 July 2005; published online 24 August 2005)

\begin{abstract}
We fabricated GaAs/AlGaAs core-shell nanowires by using selective-area metalorganic vapor phase epitaxy. First, GaAs nanowires were selectively grown on partially masked GaAs (111)B substrates; then AlGaAs was grown to form freestanding heterostructured nanowires. Investigation of nanowire diameter as a function of $\mathrm{AlGaAs}$ growth time suggested that the $\mathrm{AlGaAs}$ was grown on the sidewalls of the GaAs nanowires, forming GaAs/AlGaAs core-shell structures. Microphotoluminescence measurements of GaAs and GaAs/AlGaAs core-shell nanowires reveal an enhancement of photoluminescence intensity in GaAs/AlGaAs core-shell structures. Based on these core-shell nanowires, AlGaAs nanotubes were formed by using anisotropic dry etching and wet chemical preferential etching to confirm the formation of a core-shell structure and to explore a new class of materials. (C) 2005 American Institute of Physics. [DOI: 10.1063/1.2035332]
\end{abstract}

Recently, semiconductor nanowires are attracting great attention because of their small diameter that leads to onedimensional electron systems and because of being the possibility to be the building blocks of nanoscale electronics and photonics. The advantages of elemental (e.g., Si or Ge) ${ }^{1}$ or compound (e.g., GaAs, InP, or $\mathrm{ZnO})^{2-4}$ semiconductor nanowires compared to carbon nanotubes are in controllability of conduction types and formation of heterojunctions and superlattices. These lead to a broad range of applications such as single electron transistors, ${ }^{5}$ light emitting or laser diodes, ${ }^{6}$ and chemical sensors. ${ }^{7}$

Until now, most semiconductor nanowires are formed using catalyst-assisted vapor-liquid-solid (VLS) growth. ${ }^{8,9}$ This method is applicable to a broad range of semiconductors due to its simplicity, and ultrathin and long nanowires have been grown. Longitudinal growth to achieve abrupt doping profiles or heterojunctions has also been demonstrated by using the VLS method. ${ }^{10,11}$ However, precise control of the sites of nanowires is still difficult unless one resorts to conventional lithographic techniques to position the nanoparticle catalyst. Furthermore, incorporation of catalysts into grown crystals may deteriorate their crystalline quality.

We recently proposed and demonstrated an alternative method of fabricating semiconductor nanowires by using selective-area metalorganic vapor phase epitaxy (SA-MOVPE). ${ }^{12-14}$ Since it is a catalyst-free approach and only relies on the thermal decomposition of source materials, high crystalline quality, as well as precise controllability of the epitaxial growth can be expected. Atomic-scale abruptness in doping profiles and heterojunctions along the growth direction (vertical heterostructures) can be achieved. Furthermore, lateral growth can be controlled independently with appropriate growth conditions so that our method is also suitable for achieving heterostructured nanowires in the radial direction.

\footnotetext{
a) Author to whom correspondence should be addressed; electronic mail: nobori@rciqe.hokudai.ac.jp
}

In this letter, we describe how we grew GaAs/AlGaAs core-shell nanowires with SA-MOVPE on (111)B GaAs substrates. The formation of core-shell heterostructures is supported by observed lateral growth of AlGaAs and enhanced photoluminescence intensity in nanowires. We also fabricated freestanding $\mathrm{AlGaAs}$ nanotubes, which is another evidence for the formation of core-shell nanowires.

The growth procedure for GaAs nanowires was reported previously. ${ }^{12}$ In short, GaAs (111)B substrate partially covered with $\mathrm{SiO}_{2}$ was prepared by electron beam lithography and wet chemical etching. Then SA-MOVPE growth was carried out in a horizontal system working at 0.1 atm using trimethylgallium (TMGa), trimethylalminum (TMAl), and arsine $\left(\mathrm{AsH}_{3}\right)$.

The substrate mask pattern with an array of holes is defined in the $\mathrm{SiO}_{2}$ mask. The holes are arranged in a triangular lattice with a pitch $a$ ranging from 0.4 to $2 \mu \mathrm{m}$. For GaAs growth, the diameter $d$ of the nanowire is directly related to the opening diameter $d_{0}$. For this reason, we tried to make the holes as small as possible to obtain thin nanowires. We should note that this attempt often resulted in irregular hole sizes; however, this does not alter the main conclusion discussed here. We grew GaAs and AlGaAs in succession for core-shell heterostructures. For GaAs, the partial pressure of TMGa was $2.7 \times 10^{-7}$ atm and that of $\mathrm{AsH}_{3}$ was 5.0 $\times 10^{-4} \mathrm{~atm}$. In the growth of AlGaAs shell, the partial pressure of the total group III precursors and $\mathrm{AsH}_{3}$ were the same as for the GaAs growth. The growth temperature was $750{ }^{\circ} \mathrm{C}$ for $\mathrm{GaAs}$ and $850{ }^{\circ} \mathrm{C}$ for AlGaAs. With these conditions, perfect selective epitaxy of both GaAs and AlGaAs was achieved. The growth time was $20 \mathrm{~min}$ for GaAs and from 10 to $40 \mathrm{~min}$ for AlGaAs.

Figure 1(a) is a scanning electron microscope (SEM) image of GaAs nanowires before the growth of AlGaAs. A periodic array (pitch $1 \mu \mathrm{m}$ ) of freestanding GaAs nanowires with a height of $3 \mu \mathrm{m}$ was formed in the mask openings. The cross section of the nanowires is hexagonal with welldefined sidewalls, as is shown in the inset. Their diameters $d$ are from 50 to $100 \mathrm{~nm}$. Nonuniformity in their size origi- 


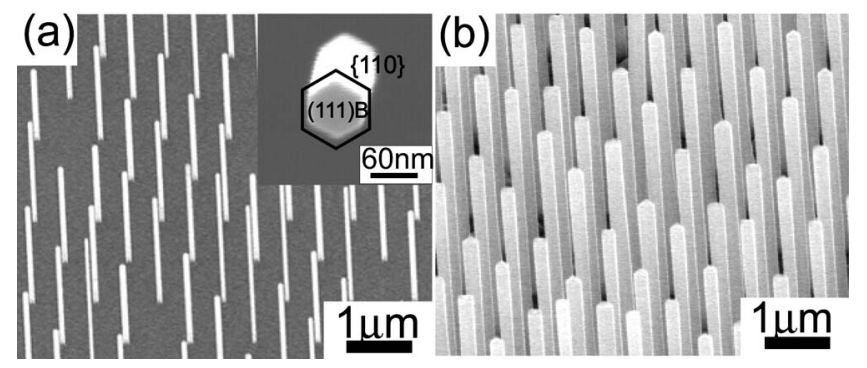

FIG. 1. (a) SEM image of GaAs nanowires. Inset shows image of nanowires from the top; (b) SEM image of nanowires after the growth of AlGaAs.

nated mainly from the irregular mask opening sizes.

Figure 1(b) shows a SEM image of AlGaAs growth on GaAs nanowires. The growth of AlGaAs was 10 min. Although they maintained hexagonal shapes, we found that the nanowires became taller $(5 \mu \mathrm{m})$ and thicker $(200-300 \mathrm{~nm})$. This indicates that AlGaAs was grown laterally on the sidewalls of GaAs nanowires as well as on their tops. This lateral growth is confirmed by the AlGaAs growth time dependence of nanowire diameter shown in Fig. 2. From these results, we conclude that freestanding GaAs/AlGaAs core-shell nanowires were formed by using SA-MOVPE. In Fig. 1(b) the thickness of the AlGaAs shells is $75-100 \mathrm{~nm}$, and the top of the nanowires is capped with $2-\mu \mathrm{m}$-thick AlGaAs. If we subtract the thickness of capped AlGaAs on the top, the aspect ratio of the core-shell nanowires is about 10 .

As reported previously, the sidewall of GaAs hexagonal nanowires are $\{110\}$ facet surfaces, vertical to the (111) plane. The appearance of these facet sidewalls is because their growth rate is slower than that of (111)B. In addition, we find that the diameter $d$ of the nanowires is equal to the opening diameter $d_{0}$ of the mask. ${ }^{14}$ This means that the growth on the $\{110\}$ sidewalls is negligible for GaAs grown under the present conditions.

On the other hand, the growth takes place on the sidewall surfaces in the lateral direction as well as on the top surfaces for AlGaAs. Ando et al. have reported an enhanced lateral growth of AlGaAs on the $\{110\}$ sidewalls of selectively grown GaAs wire structures on (111)B in slightly different conditions. ${ }^{15}$ The enhanced lateral growth on the sidewall $\{110\}$ facet in AlGaAs could be explained by a stronger bonding and a lower migration length of $\mathrm{Al}$ atoms on $\{110\}$ surfaces.

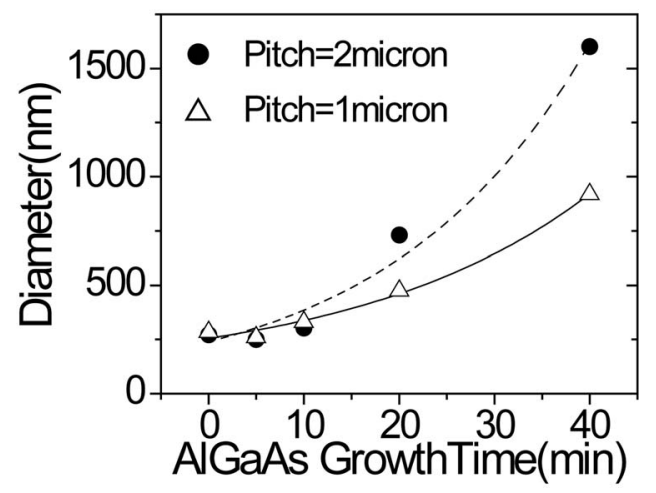

FIG. 2. AlGaAs growth time dependence of nanowire thickness. Initial GaAs nanowire diameter was $270 \mathrm{~nm}$. Nanowire diameter increased with

AlGaAs growth time.
Downloaded 23 Jan 2006 to 133.87 .26 .100 . Redistribution subject to AlP license or copyright, see http://apl.aip.org/apl/copyright.jsp

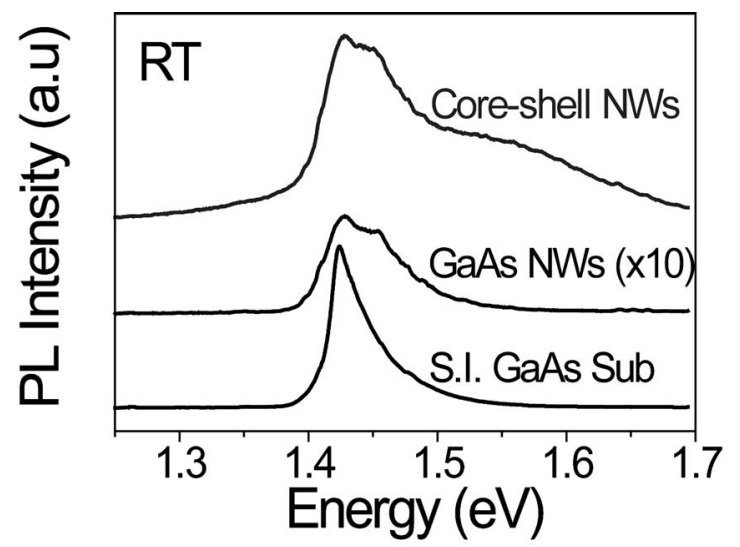

FIG. 3. PL spectra of core-shell nanowires (NWs), bare GaAs nanowires, and reference semi-insulating GaAs substrate. Intensity of core-shell nanowires is about 20 times larger that of bare GaAs nanowires.

To obtain the results given in Fig. 2, we conducted an experiment of growing GaAs nanowires with a substrates mask with larger holes and longer hole pitches to minimize the effect of irregular hole sizes. In this experiment, the obtained diameter of GaAs nanowires was $270 \mathrm{~nm}$ and their typical size fluctuation was on the order of several percent. ${ }^{12}$ We find that the amount of AlGaAs lateral growth is nonlinear and depends on the pitch $a$ of holes. Furthermore, as mentioned earlier, the amount of lateral growth for a 10 -min growing period was estimated to be $75-100 \mathrm{~nm}$ for nanowires when $d=50-80 \mathrm{~nm}$, whereas it was negligible in the results presented in Fig. 2. These findings mean that the lateral growth rate of $\mathrm{AlGaAs}$ is critically dependent on the size and arrangement of nanowires. Further studies are needed to clarify the detail of the growth process.

Next, we conducted microphotoluminescence measurement of GaAs and GaAs/AlGaAs nanowire arrays at $290 \mathrm{~K}$. In this experiment, a $\mathrm{He}-\mathrm{Ne}$ laser was used for excitation and the excitation intensity was $60 \mu \mathrm{W}$. Figure 3 shows the photoluminescence (PL) spectra of GaAs and core-shell nanowires. The average size of nanowires is $80 \mathrm{~nm}$ for GaAs, and $300 \mathrm{~nm}$ for the core shell, and the pitch of the array is $0.4 \mu \mathrm{m}$. For reference, the PL spectrum of semiinsulating (SI) GaAs substrate is also plotted.

We can see some differences between the two types of nanowires and the reference. First, the PL intensity of coreshell nanowires was much stronger than that of bare GaAs nanowires by a factor of about 20, and almost equal to that of the SI GaAs substrate. Weak emission of GaAs nanowires can be explained by nonradiative recombination of photoexcited carriers at the air-exposed GaAs sidewall surface where high density of surface states exists. The recovery of PL intensity in core-shell structures is also explained by the surrounding AlGaAs barrier that prevents the coupling of photoexcited carriers in GaAs with the surface states. ${ }^{16,17}$ These results are consistent with our previous reports on GaAs/AlGaAs nanowires, ${ }^{12}$ and reveal a clue to obtaining high-quality and optically active nanowires.

Second, in comparing the PL spectra of GaAs nanowires, GaAs/AlGaAs core-shell nanowires, and SI GaAs substrate, we attribute emissions at $1.45 \mathrm{eV}$ in both nanowires to GaAs, show a blueshift of $30 \mathrm{meV}$ from emissions of the GaAs bulk. In circular nanowires surrounded with infinitely high potential barriers, the ground-state quantized energy associated with lateral confinement is given by $2 \hbar^{2} \lambda_{01}^{2} / m d^{2}$, AIP license or copyright, see http://apl.aip.org/apl/copyright.jsp 


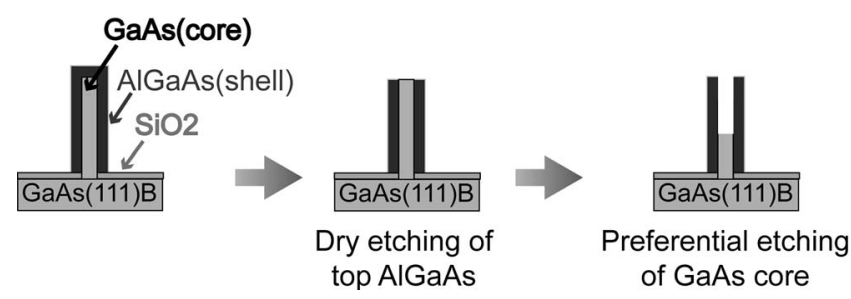

FIG. 4. Fabrication processes for AlGaAs nanotubes. Starting from coreshell nanowires, top AlGaAs was removed by dry etching, followed by preferential wet etching to remove GaAs core.

where $m$ is the effective mass and $\lambda_{01}$ is the first zero of the zeroth order Bessel function. For a GaAs core of $80 \mathrm{~nm}$, the expected blueshift is only $2.5 \mathrm{meV}$, much smaller than the observed $30 \mathrm{meV}$. Possible origin of the shift may be $\operatorname{strain}^{18}$ or other mechanisms. A more careful study is required to clarify the origin of the blueshift of PL peaks in nanowires.

Third, when we compared the PL spectrum of the two types of nanowires, a broad feature at the high-energy side of GaAs is observed in core-shell nanowires. We think this is an emission from the AlGaAs shell and its broadening indicates a broad $\mathrm{Al}$ composition distribution. Estimating from the high-energy shoulder of the spectrum shows the aluminum content is distributed approximately from $4 \%$ to $16 \%$, while $\mathrm{PL}$ and $\mathrm{x}$-ray diffraction measurement of AlGaAs grown on planar (111)B shows the Al content to be $12 \%$. The origin of the tendency of slightly smaller $\mathrm{Al}$ incorporation in nanowires is probably due to a difference in the migration length between Ga and Al during SA-MOVPE. Furthermore, as we reported previously, since the growth rate of GaAs depends on the diameter of the nanowires, a probability exists that the growth rate and $\mathrm{Al}$ incorporation rate in $\mathrm{AlGaAs}$ changes as the diameter increases due to lateral growth. This could give rise to nonuniform aluminum content in nanowires. In addition, the compositions of aluminum in $\mathrm{AlGaAs}$ between the sidewalls and that on top surfaces are possibly different.

Finally, we fabricated AlGaAs nanotubes out of coreshell nanowires. For this, we carried out a two-step etching process: specifically, anisotropic dry etching followed by preferential wet etching on core-shell nanowires, as described in Fig. 4. For the dry etching, we used reactive ion beam etching (RIBE) with $\mathrm{CH}_{4}, \mathrm{H}_{2}, \mathrm{Ar}$, and $\mathrm{N}_{2}$ for $30 \mathrm{~min}$, and used a mixture of $\mathrm{NH}_{4} \mathrm{OH}$ and $\mathrm{H}_{2} \mathrm{O}_{2}$ for wet etching.

The result of the two-step etching is given in Fig. 5; we obtained freestanding AlGaAs nanotubes. As is shown in

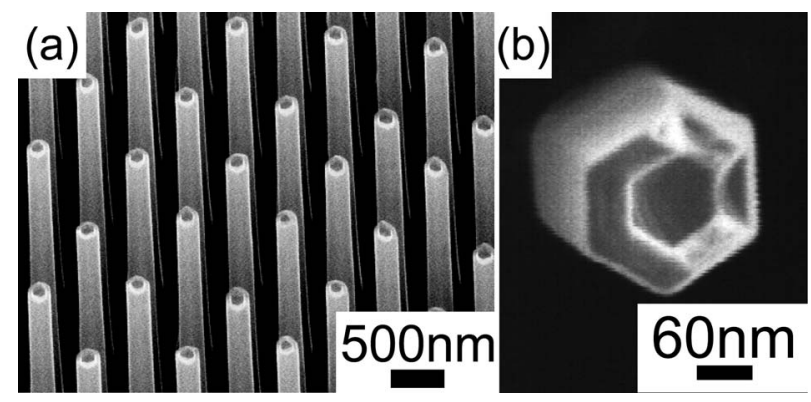

FIG. 5. (a) SEM images of freestanding AlGaAs nanotubes. Their inner and outer diameters are 100 and $200 \mathrm{~nm}$, respectively. (b) Top view SEM image of AlGaAs nanotube.
Fig. 5(b), the nanotubes remained hexagonal in their outer and inner part. The length of AlGaAs nanotubes is $3 \mu \mathrm{m}$, and their inner and outer diameters are 100 and $200 \mathrm{~nm}$, respectively. This indicates that the thickness of the AlGaAs layer is $50 \mathrm{~nm}$. These results are consistent with the results of SEM measurement shown Fig. 1.

We think the somewhat smaller outer diameter and tapering in the nanotubes originated from side etching of AlGaAs during RIBE or from imperfect selectivity in the wet etching process. The selectivity of GaAs and AlGaAs with present $\mathrm{NH}_{4} \mathrm{OH} / \mathrm{H}_{2} \mathrm{O}_{2}$ mixture is usually several tens to hundreds, ${ }^{19}$ and could be improved by optimization of the etching. Note that these nanotubes are another proof of the formation of core-shell nanowires from SA-MOVPE and simple etching processes. We should also mention that our technology for fabricating nanotubes can be applied to other semiconductors with appropriate choices of dry etching gas and conditions, and of the solution for wet etchant. Thus, our approach is a promising one for exploring a new class of nanoscale materials.

The authors thank their fruitful discussions with Professor Hideki Hasegawa, Professor Tamotu Hashizume, Professor Charles Tu, Dr. Tutomu Muranaka, and Dr. Premila Mohan, and the experimental support of Noboru Ooike and Akira Koike. This work is financially supported in part by a Grant-in-Aid for Scientific Research provided by the Japan Society for Promotion of Science.

${ }^{1}$ L. J. Lauhon, M. S. Gudiksen, D. Wang, and C. M. Lieber, Nature (London) 420, 57 (2002).

${ }^{2}$ B. J. Ohlsson, M. T. Bjork, M. H. Magnusson, K. Deppert, and L. Samuelson, Appl. Phys. Lett. 79, 3335 (2001).

${ }^{3}$ Y. Watanabe, S. Bhunia, S. Fujikawa, T. Kawamura, H. Nakashima, K. Furukawa, and K. Torimitsu, Thin Solid Films 464-465, 248 (2004).

${ }^{4}$ P. Yang, H. Yan, S. Mao, R. Russo, J. Johnshon, R. Saykally, N. Morris, J. Pham, R. He, and H. Choi, Adv. Funct. Mater. 5, 323 (2002).

${ }^{5}$ C. Thelander, T. Martensson, M. T. Bjork, B. J. Ohlsson, M. W. Larsson, L. R. Wallenberg, and L. Samuelson, Appl. Phys. Lett. 83, 2052 (2003).

${ }^{6}$ X. Duan, Y. Huang, R. Agarwal, and C. M. Lieber, Nature (London) 421, 241 (2003).

${ }^{7}$ D. Zhang, C. Li, X. Liu, S. Han, T. Tang, and C. Zhou, Appl. Phys. Lett. 83, 1845 (2003).

${ }^{8}$ R. S. Wagner and W. C. Ellis, Appl. Phys. Lett. 4, 89 (1964).

${ }^{9}$ K. Hiruma, M. Yazawa, T. Katsuyama, K. Ogawa, K. Haraguchi, M. Koguchi, and H. Kakibayashi, J. Appl. Phys. 77, 447 (1995).

${ }^{10}$ M. T. Bjork, B. J. Ohlsson, T. Sass, A. I. Persson, C. Thelander, M. H. Magunusson, K. Deppert, L. R. Wallenberg, and L. Samuelson, Appl. Phys. Lett. 80, 1058 (2002).

${ }^{11}$ L. Samuelson, M. T. Bjork, K. Deppert, M. Larsson, B. J. Ohlsson, N. Panev, A. I. Persson, N. Skold, C. Thelander, and L. R. Wallenberg, Physica E (Amsterdam) 21, 560 (2004).

${ }^{12}$ J. Motohisa, J. Takeda, M. Inari, J. Noborisaka, and T. Fukui, Physica E (Amsterdam) 23, 298 (2004).

${ }^{13}$ J. Motohisa, J. Noborisaka, J. Takeda, M. Inari, and T. Fukui, J. Cryst. Growth 272, 180 (2004).

${ }^{14}$ J. Noborisaka, J. Motohisa, and T. Fukui, Appl. Phys. Lett. 86, 213102 (2005).

${ }^{15}$ S. Ando, S. S. Chan, and T. Fukui, J. Cryst. Growth 115, 69 (1991).

${ }^{16}$ S. Kodama, S. Koyanagi, T. Hashizume, and H. Hasegawa, J. Vac. Sci. Technol. B 13, 1794 (1995).

${ }^{17}$ J. M. Moison, K. Elcess, F. Houzay, J. Y. Marzin, J. M. Gerard, F. Barthe, and M. Bensoussan, Phys. Rev. B 41, 12945 (1990).

${ }^{18}$ H. W. Seo, S. Y. Bae, and J. Park, J. Chem. Phys. 116, 9492 (2002).

${ }^{19}$ T. Kitano, S. Izumi, H. Minami, T. Ishikawa, K. Sato, T. Sonoda, and M. Otsubo, J. Vac. Sci. Technol. B 15, 167 (1996). 